\title{
Asymptotic Analysis of Reliability for Switching Systems in Light and Heavy Traffic Conditions
}

\author{
Vladimir V. Anisimov \\ Bilkent University, Ankara, Turkey $\&$ Kiev University, Kiev, Ukraine
}

\begin{abstract}
An asymptotic analysis of flows of rare events switched by some random environment is provided. An approximation by nonhomogeneous Poisson flows in case of mixing environment is studied. Special notions of $S$-set and "monotone" structure for finite Markov environment are introduced. An approximation by Poisson flows with Markov switches in case of asymptotically consolidated environment is proved. An analysis of the 1st exit time from a subset is also given. In heavy traffic conditions an averaging principle for trajectories with Poisson approximation for flows of rare events in systems with fast switches is proved. The method of proof is based on limit theorems for processes with semi-Markov switches.

Applications to the reliability analysis of state-dependent Markov and semiMarkov queueing systems in light and heavy traffic conditions are considered

Keywords and phrases: asymptotic analysis, reliability, switching processes, rare events, Markov and semi-Markov processes, consolidation, queueing models, light and heavy traffic.
\end{abstract}

\subsection{Introduction}

Models of real technical systems have usually a high dimension and a complex structure. Exact analytic solutions can be obtained only for special rare cases. Therefore asymptotic methods play a basic role in the investigation and approximate modelling. At the analysis of highly reliable complex systems various events connected with failures, changes in the regime of operation, exceeding of some level, etc., usually have small probabilities (or rates) and depend on a trajectory of a system. This implies a significance of the analysis of so called 
flows of rare events in reliability theory. Different asymptotic approaches for reliability analysis of various classes of stochastic systems are studied in the books [Korolyuk and Turbin (1978), Kovalenko (1980), Anisimov et al. (1987), Anisimov (1988)]. An asymptotic analysis of wide classes of regenerative queueing models is considered by Soloviev (1970). A survey of results devoted to the analysis of rare events in queueing systems is given by Kovalenko (1994).

In this paper we provide the asymptotic analysis of flows of rare events switched by some random environment. The environment may be nonhomogeneous in time and not regenerative. In case when the environment satisfies an asymptotically mixing condition, an approximation by nonhomogeneous Poisson flows is proved. A special attention is given to the case of finite Markov processes $(M P)$ with transition rates of different orders. The notions of $S$ set and "monotone" structure are introduced in non-homogeneous case and an asymptotic behaviour of a flow of rare events on $S$-sets and the exit time from the $S$-set are studied. We mention that in homogeneous case corresponding notions were introduced by Anisimov (1970),(1973),(1974).

The method of $S$-sets allows to study the asymptotic behaviour of the time of 1st loss of a call for wide classes of queueing systems and networks with finite number of states and in the case of fast service or light loading (see Anisimov et al. (1987), Anisimov and Sztrik (1989), Sztrik and Kouvatsos (1991), Sztrik (1992), Anisimov (1996)).

In case when the environment is a non-homogeneous $M P$ which satisfies conditions of the asymptotic consolidation (aggregation) of a state space, an approximation of flows of rare events by Poisson flows with Markov switches is studied.

We mention that models of the asymptotic consolidation of the state space of $M P$ 's and semi-Markov processes $(S M P)$ and algorithms of the sequential aggregation of the state space are studied in Anisimov (1970), (1973), (1974). For homogeneous MP's in continuous time with transition rates of different orders similar results (an analysis of stationary and transient probabilities in case of the asymptotic aggregation of the state space) are obtained by Courtois (1977) and Bobbio and Trivedi (1985). On the base of operator technique models of the asymptotic consolidation of homogeneous MP and SMP's are studied by Korolyuk and Turbin (1978).

In heavy traffic conditions an averaging principle for trajectories with Poisson approximation for flows of rare events is proved. The method of proof is based on the results of Anisimov (1994), (1995) and Anisimov and Aliev (1992) for processes with semi-Markov switches.

Applications to the reliability analysis of state-dependent Markov and semiMarkov queueing systems of the type $M_{S M, Q} / M_{S M, Q} / m / k$ in light and heavy traffic conditions are considered. The models of the asymptotic consolidation and the case of highly reliable servers are considered as well. Another applications can be found in Anisimov (1996), (1998), (1999). 
The results obtained give us an approximate analytic approach in modelling of reliability characteristics of rather complex queueing models in transient and stable regimes under light and heavy traffic conditions.

\subsection{Flows of Rare Events in Systems with Mixing}

In various models the analysis of reliability is essentially connected with analysis of flows of rare events on the trajectory of a system. In many models rare events may appear only in some region of the state space and in some cases this region is accessible with small probability.

Let for any $n>0 \quad x_{n}(t), t \geq 0$ be a random process with state space $X$ and $\left\{q_{n}(x, t), x \in X, t \geq 0\right\}$ be nonnegative functions such that $q_{n}(x, t)=0$ as $x \notin Z_{n}$ where $Z_{n} \subset X$. Denote by $\left(x_{n}(t), \Pi_{n}(t)\right), t \geq 0$ a two-component process such that $\Pi_{n}(t)$ is a Poisson process switched by $x_{n}(t)$ with instantaneous rate of jump at time $t q_{n}\left(x_{n}(t), t\right)$. We can interpret $q_{n}(x, t)$ as the rate of failure in state $x$ at time $t$, and $\Pi_{n}(t)$ is the total number of failures on the interval $[0, t]$. We study the behaviour of $\Pi_{n}(t)$. In this section we suppose that $x_{n}(t)$ satisfies an asymptotically mixing condition in $Z_{n}$.

Let us introduce a strong mixing coefficient (s.m.c.) in $Z_{n}$ :

$$
\begin{gathered}
\varphi_{n}\left(u, Z_{n}\right)=\sup _{t \geq 0} \sup _{A_{1}, A_{2} \subset Z_{n}} \mid \mathbf{P}\left\{x_{n}(t) \in A_{1}, x_{n}(t+u) \in A_{2}\right\}- \\
\quad \mathbf{P}\left\{x_{n}(t) \in A_{1}\right\} \mathbf{P}\left\{x_{n}(t+u) \in A_{2}\right\} \mid .
\end{gathered}
$$

Put

$$
\Lambda_{n}(t)=\mathbf{E} \int_{0}^{V_{n} t} q_{n}\left(x_{n}(v), v\right) \mathrm{d} v,
$$

where $V_{n} \rightarrow \infty$, and let $\widehat{\Pi}_{n}(t)$ be a nonhomogeneous Poisson process with integral intensity $\Lambda_{n}(t)$ that is

$$
\mathbf{E} \exp \left\{i \theta \widehat{\Pi}_{n}(t)\right\}=\exp \left\{\left(e^{i \theta}-1\right) \Lambda_{n}(t)\right\} .
$$

For some fixed $T>0$ denote $q_{n}=\sup _{t \in[0, T]} \sup _{x \in Z_{n}} q_{n}(x, t)$.

Theorem 8.1 Let

$$
\alpha_{n}=V_{n} q_{n}^{2} \int_{0}^{V_{n} T} \varphi_{n}\left(u, Z_{n}\right) \mathrm{d} u \rightarrow 0 .
$$

Then the finite-dimensional distributions of processes $\Pi_{n}\left(V_{n} t\right)$ and $\widehat{\Pi}_{n}(t)$ on the interval $[0, T]$ are asymptotically equivalent. 
Proof. According to the construction of $\Pi_{n}(\cdot)$, for any $t>0$ we have a representation

$$
\left.\mathbf{E} \exp \left\{-\theta \Pi_{n}\left(V_{n} t\right)\right)\right\}=\mathbf{E} \exp \left\{\left(e^{-\theta}-1\right) \int_{0}^{V_{n} t} q_{n}\left(x_{n}(v), v\right) \mathrm{d} v\right\} .
$$

Taking into account the inequality

$$
\left|\int_{Y} f(y) P(\mathrm{~d} y)-\int_{Y} f(y) Q(\mathrm{~d} y)\right| \leq \sup _{y \in Y} f(y) \sup _{A \in \mathcal{B}_{Y}}|P(A)-Q(A)|
$$

which is true for any non-negative bounded function $f(y)$ and any non-negative measures $P(\cdot), Q(\cdot)$ on $Y$, we easily get that $\mid \mathbf{E} q_{n}\left(x_{n}(u), u\right) q_{n}\left(x_{n}(v), v\right)$ $\mathbf{E} q_{n}\left(x_{n}(u), u\right) \mathbf{E} q_{n}\left(x_{n}(v), v\right) \mid \leq q_{n}^{2} \varphi_{n}\left(v-u, Z_{n}\right)$ as $u<v$. Then using the inequality $\left|e^{-a}-e^{-b}\right| \leq|a-b|, a, b>0$, due to (2.2) we get

$$
\begin{gathered}
\left.\mid \mathbf{E} \exp \left\{-\theta \Pi_{n}\left(V_{n} t\right)\right)\right\}-\exp \left\{\left(e^{-\theta}-1\right) \Lambda_{n}(t)\right\} \mid \\
\leq\left|e^{-\theta}-1\right|^{2}\left(\mathbf{E}\left(\int_{0}^{V_{n} t} q_{n}\left(x_{n}(v), v\right) \mathrm{d} v-\Lambda_{n}(t)\right)^{2}\right)^{1 / 2}=O\left(\sqrt{\alpha}_{n}\right) \rightarrow 0 .
\end{gathered}
$$

This relation proves the equivalence of one-dimensional distributions of $\Pi_{n}\left(V_{n} t\right)$ and $\widehat{\Pi}_{n}(t)$. By analogy the equivalence of finite-dimensional distributions can be proved.

Remark 8.1 In particular if $q_{n}=O\left(1 / V_{n}\right)$, then (2.2) is satisfied if there exists such nonrandom sequence $r_{n}$ that as $n \rightarrow \infty$

$$
V_{n}^{-1} r_{n} \rightarrow 0, \quad \sup _{u>r_{n}} \varphi_{n}(u) \rightarrow 0
$$

Remark 8.2 Let (2.2) hold and there exist a continuous function $\Lambda_{0}(t)$ such that for any $t \in[0, T]$

$$
\lim _{n \rightarrow \infty} \Lambda_{n}(t)=\Lambda_{0}(t)
$$

Then the sequence of processes $\Pi_{n}\left(V_{n} t\right) J$-converges on $[0, T]$ to the Poisson process $\Pi_{0}(t)$ with integral intensity $\Lambda_{0}(t)$ (J-convergence means the convergence of measures in Skorokhod space $\left.\mathcal{D}_{T}\right)$.

This means that flows of rare events in systems with mixing can be approximated by Poisson processes with average integral intensity. Denote now by $\nu_{n}$ the time of the first jump of $\Pi_{n}(t)$ (time of first failure).

Consequence 8.1 If $Q(a)=\left(a^{2} / 2\right)^{-1}\left(e^{a}-1-a\right)$ and $t<T$, then

$$
\exp \left\{-\Lambda_{n}(t)\right\} \leq \mathbf{P}\left\{\nu_{n}>V_{n} t\right\} \leq \exp \left\{-\Lambda_{n}(t)\right\}\left(1+\frac{1}{2} Q\left(\Lambda_{n}(t)\right) \alpha_{n}\right)
$$


Analogous results can be obtained for discrete time models.

The main problem in applications is how to estimate the function $\Lambda_{n}(\cdot)$ and the s.m.c. $\varphi_{n}\left(u, Z_{n}\right)$. Further we consider homogeneous and nonhomogeneous finite Markov processes and show that $\Lambda_{n}(\cdot)$ can be replaced by an equivalent function calculated with the help of stationary or quasi-stationary distribution.

\subsection{Asymptotically Connected Sets ( $S$-Sets)}

We introduce an important notion of $V_{n}$-S -set (asymptotically connected set). Let $x_{n}(t), t \geq 0$ be a Markov process $(M P)$ in discrete or continuous time with finite state space $X=\{1,2, \ldots, r\}$. Let $X_{0}$ be some fixed subset of $X$.

Definition 8.1 The subset $X_{0}$ is called a $V_{n}$-S-set if as $n \rightarrow \infty$ $\mathbf{P}\left\{x_{n}(t) \in X_{0}, t \leq V_{n} / x_{n}(0)=i\right\} \rightarrow 1$ and for any $i, j \in X_{0}$

$$
\mathbf{P}\left\{\text { there exists } u, u<V_{n} \text { such that } x_{n}(u)=j / x_{n}(0)=i\right\} \rightarrow 1 .
$$

This means that on the interval $\left[0, V_{n}\right]$ process stays in $X_{0}$ with probability close to one and all states in $X_{0}$ asymptotically communicate. In particular the total state space $X$ may form a $V_{n}$-S-set. In that case (2.3) is satisfied.

\subsubsection{Homogeneous case}

Consider now discrete time and suppose that $x_{n k}, k \geq 0$ is a homogeneous $M P$ with finite state space $X$. Let $\left\{\chi_{n k}(i), i \in X\right\}, k \geq 0$ be jointly independent families of rare indicators, that is $\mathbf{P}\left(\chi_{n k}(i)=1\right)=1-\mathbf{P}\left(\chi_{n k}(i)=0\right)=q_{n}(i)$, where $q_{n}(i) \rightarrow 0, i \in X$. Let

$$
\Pi_{n}\left(V_{n} t\right)=\sum_{k=0}^{\left[V_{n} t\right]} \chi_{n k}\left(x_{n k}\right) .
$$

Suppose that $X$ forms a $V_{n}$-S -set. Denote by $\pi_{n}(i), i \in X$ the stationary distribution of $x_{n k}$ which exists at this assumption. Put $\Lambda_{n}=\sum_{i \in X} \pi_{n}(i) q_{n}(i)$.

Statement 8.1 If $\limsup _{n \rightarrow \infty} V_{n} \Lambda_{n}<\infty$, then $V_{n} \pi_{n}(i) \rightarrow \infty, i \in X$, and finite-dimensional distributions of the process $\Pi_{n}\left(V_{n} t\right)$ and the Poisson process with parameter $V_{n} \Lambda_{n}$ are asymptotically equivalent.

These results give also the possibility to study the exit time from the region. Let $X_{0}$ be some fixed subset of $X$. Denote by $\nu_{n}\left(i, X_{0}\right)$ the exit time from $X_{0}$ given that the initial state is $i \in X_{0}$. 
Definition 8.2 The subset $X_{0}$ is called an $S$-set if for any $i, j \in X_{0}$

$\mathbf{P}\left\{\right.$ there exists $k, k<\nu_{n}\left(i, X_{0}\right)$ such that $\left.x_{n k}=j / x_{n 0}=i\right\} \rightarrow 1$ as $n \rightarrow \infty$.

Consider an auxiliary $M P \tilde{x}_{n k}$ with state space $X_{0}$ and matrix of transition probabilities $\tilde{P}_{n}\left(X_{0}\right)=\left\|p_{n}(i, j) p_{n}\left(i, X_{0}\right)^{-1}\right\|, i, j \in X_{0}$, where $p_{n}\left(i, X_{0}\right)=$ $\sum_{l \in X_{0}} p_{n}(i, l)$. Denote by $\tilde{\pi}_{n}(i), i \in X_{0}$ its stationary distribution (which exists at least at large enough $n)$ and put $g_{n}\left(X_{0}\right)=\sum_{i \in X_{0}} \tilde{\pi}_{n}(i)\left(1-p_{n}\left(i, X_{0}\right)\right)$.

Statement 8.2 If $X_{0}$ forms an $S$-set, then for any $i_{0} \in X_{0}$

$$
\lim _{n \rightarrow \infty} \mathbf{P}\left\{g_{n}\left(X_{0}\right) \nu_{n}\left(i_{0}, X_{0}\right)>t\right\}=\exp \{-t\}, t>0
$$

We mention that $X_{0}$ forms also a $g_{n}\left(X_{0}\right)^{-1}-S$-set for $\tilde{x}_{n k}$ and it is always possible to find such $V_{n}$ that $V_{n} g_{n}\left(X_{0}\right) \rightarrow 0$ and $X_{0}$ forms a $V_{n}-S$-set for $x_{n k}$.

Let us consider a special type of $V_{n}-S$-set which is called a "monotone structure". We give here a corresponding definition for continuous time. The case of discrete time was considered in Anisimov at al. (1987), Anisimov (1996).

Let $x_{n}(t), t \geq 0$ be some $M P$ with finite state space $Z$ which can be represented in the form: $Z=\left\{(i, s), i \in X_{s}, s=\overline{0, r}\right\}$, and given transition rates $\mu_{n}((i, s),(j, q))$.

Definition 8.3 The state space $Z$ is called a "monotone structure" if as $n \rightarrow$ $\infty$ the following asymptotic relations hold:

1. $\mu_{n}((i, s),(j, s+1))=\varepsilon_{n}(s) a_{i j}(s)(1+o(1)), i \in X_{s}, j \in X_{s+1}$, where $\varepsilon_{n}(s) \rightarrow 0, s=\overline{0, r-1}$;

2. $\mu_{n}((i, s),(j, s+k))=0, i \in X_{s}, j \in X_{s+k}, s=\overline{0, r-2}, k>1$;

3. $\mu_{n}((i, s),(j, k))=\mu_{i j}(s, k)(1+o(1)), i \in X_{s}, j \in X_{k}, s=\overline{0, r}, k \leq s$;

4. the matrix $G(s)-M(s)$ is invertible for each $s=\overline{1, r}$, where $G(s)$ is a diagonal matrix with elements $\mu_{i}^{(s)}=\sum_{m \leq s, j \in X_{m}} \mu_{i j}(s, m)$ and $M(s)=\left\|\mu_{i j}(s, s)\right\|, i, j \in X_{s}, i \neq j$, where we put $\mu_{i i}(s, s) \equiv 0, i \in X_{s}$;

5. an auxiliary MP with state space $\left\{(i, 0), i \in X_{0}\right\}$ and transition rates $\mu_{i j}(0,0)$ is irreducible with stationary distribution $\rho_{i}, i \in X_{0}$.

We call a subset of states $Z_{q}=\left\{(i, q), i \in X_{q}\right\}$ a $q$-level, $q=\overline{0, r}$.

Let $\bar{\rho}_{n}(s)=\left(\rho_{n}(i, s), i \in X_{s}\right), s=\overline{0, m}$, and $\bar{\rho}=\left(\rho_{i}, i \in X_{s}\right)$ be rowvectors, where $\rho_{n}(i, s)$ be the stationary probability of state $(i, s)$. We put $\delta_{n}(s)=\prod_{j=0}^{s-1} \varepsilon_{n}(j)$. 
Theorem 8.2 If $Z$ forms a monotone structure, then for any $v_{n} \rightarrow \infty$ it also forms $v_{n} \delta_{n}(r)^{-1}$-S-set and for $q=\overline{1, r}$ the following representation holds:

$$
\bar{\rho}_{n}(q)=\delta_{n}(q) \bar{\alpha}(q)(1+o(1))
$$

where $\bar{\alpha}(q)=\bar{\rho} \prod_{j=0}^{q-1} A(j)(G(j+1)-M(j+1))^{-1}, A(s)=\left\|a_{i j}(s)\right\|, i, j \in X_{s}$, $\prod_{j=k}^{s} C(j)=C(k) C(k+1) \cdots C(s)$.

The proof is made recursively to the order of the monotone structure by analogy to discrete case [Anisimov (1996)].

Remark 8.3 If $Z$ forms a monotone structure, then for any level $q$ and some $0<a<1 \quad \varphi_{n}\left(u, Z_{q}\right) \leq C \delta_{n}(q) a^{u}$.

Using Remark 8.3 we can study flows of rare events of different orders on the monotone structure. Denote by $\Pi_{n}(t)$ a switched Poisson process constructed on the trajectory of $x_{n}(t)$ according to the rate $q_{n}(i, s)$ in state $(i, s)$, where $q_{n}(i, s)=q_{n} b_{i}(s) \prod_{j=s}^{r-1} \varepsilon_{n}(j)(1+o(1))$ and $q_{n} \rightarrow 0$ (we set $\prod_{r}^{r-1}=1$ ). Put $V_{n}=\left(q_{n} \delta_{n}(r)\right)^{-1}$.

Consequence 8.2 If the state space $Z$ forms a monotone structure, then $\Pi_{n}\left(V_{n} t\right)$ $J$-converges to the Poisson process with parameter $A=\sum_{s=0}^{r}(\bar{\alpha}(s), \bar{b}(s))$, where $\bar{b}(s)$ is a column vector with elements $b_{i}(s), i \in X_{s}$.

In particular if $Z$ is a subset of the state space and the rate of exit from state $(i, s)$ is equal to $q_{n}(i, s)$, then using Consequence 8.2 we get an exponential approximation with parameter $A$ for the variable $V_{n}^{-1} \Omega_{n}(i, s)$, where $\Omega_{n}(i, s)$ is the exit time from $Z$ starting from state $(i, s)$.

These results can be extended to the case when $x_{n}(t), t \geq 0$ is a $S M P$ such that an embedded $M P$ forms a monotone structure [Anisimov (1996)].

\subsubsection{Nonhomogeneous case}

We extend now the notion of the monotone structure to the case when $x_{n}(t)$ is a nonhomogeneous $M P$. Suppose that $x_{n}(t)$ takes values in $Z=\{(i, s), i \in$ $\left.X_{s}, s=\overline{0, r}\right\}$ and transition rates at time $t$ are $\mu_{n}((i, s),(j, q), t)$.

Let there exist a normalizing factor $V_{n}$ such that the rates $\mu_{n}\left((i, s),(j, q), V_{n} t\right)$ satisfy all items of Definition 8.3 where the values $a_{i j}(s)=a_{i j}(s, t)$ and $\mu_{i j}(s, k)=$ $\mu_{i j}(s, k, t)$ depend on $t$. That means for instance $\mu_{n}\left((i, s),(j, q), V_{n} t\right)=\varepsilon_{n}(s) a_{i j}(s, t)(1+$ $o(1)$ for item 1 . We denote corresponding matrices as $G(s, t)$ and $M(s, t)$. Let $\rho_{i}(t), i \in X_{0}$ be the stationary distribution of auxiliary $M P$ with state space $\left\{(i, 0), i \in X_{0}\right\}$ and transition rates $\mu_{i j}(0,0, t), i \neq j$.

Suppose also that the following condition is satisfied: functions $a_{i j}(s, t)$ and $\mu_{i j}(s, k, t)$ are piecewise continuous in $t$ and if $a_{i j}(s, 1)>0$, then for some $c_{0}>0$ $a_{i j}(s, t) \geq c_{0}$ on some interval $[0, T]$ (the same for $\mu_{i j}(s, k, t)$ ). 
Then the set $Z$ forms a monotone structure in the scale of time $V_{n}$.

Denote

$$
\bar{\alpha}(q, t)=\bar{\rho}(t) \prod_{j=0}^{q-1} A(j, t)(G(j+1, t)-M(j+1, t))^{-1} .
$$

Theorem 8.3 If $Z$ forms a monotone structure, then as $q=\overline{1, r}, 0<t<T$

$$
\left(\mathbf{P}\left\{x_{n}\left(V_{n} t\right)=(i, q)\right\}, i \in X_{q}\right)=\delta_{n}(q) \bar{\alpha}(q, t)(1+o(1)),
$$

and

$$
\mathbf{P}\left\{x_{n}\left(V_{n} t\right)=(i, 0)\right\}=\rho_{i}(t)(1+o(1)), i \in X_{0} .
$$

We mention that right hand side plays a role of quasi-stationary probability.

Using this result we can study the behaviour of flows of rare events by analogy to Consequence 8.2. Let $\Pi_{n}(t)$ be constructed on $x_{n}(t)$ according to the rate $q_{n}(i, s, t)$, where $q_{n}\left(i, s, V_{n} t\right)=q_{n} b_{i}(s, t) \prod_{j=s}^{r-1} \varepsilon_{n}(j)$ and $V_{n}=\left(q_{n} \delta_{n}(r)\right)^{-1}$.

Consequence 8.3 If $Z$ forms a monotone structure, then $\Pi_{n}\left(V_{n} t\right)$ converges to the Poisson process with local rate $\hat{\lambda}(t)=\sum_{s=0}^{r}(\bar{\alpha}(s, t), \bar{b}(s, t))$, where $\bar{b}(s, t)$ is a vector with elements $b_{i}(s, t), i \in X_{s}$.

In particular if $Z$ is a subset of the state space and the rate of exit from state $(i, s)$ at time $t$ is equal to $q_{n}(i, s, t)$, then

$$
\mathbf{P}\left\{V_{n}^{-1} \Omega_{n}(i, s)>t\right\} \rightarrow \exp \left\{-\int_{0}^{t} \hat{\lambda}(u) \mathrm{d} u\right\}
$$

\subsection{Asymptotically Consolidated Systems}

We note that as it follows from Consequences 8.2, 8.2 the asymptotic behaviour of exit time from $S$-set does not depend on the initial state. This gives the possibility of studying models of asymptotic consolidation of state space (see Anisimov, 1973, 1978, 1998, Anisimov et al., 1987).

Let $x_{n}(t)$ be a nonhomogeneous $M P$ with finite state space $X$ and transition rates $\mu_{n}(i, j, t), i, i \in X, i \neq j, t \geq 0$. Suppose that for $X$ and $\mu_{n}(i, j, t)$ we have the following representation:

$$
\begin{gathered}
X=\bigcup_{k \in Y} X_{k}, \text { where } X_{k_{1}} \bigcap X_{k_{2}}=\emptyset \text { as } k_{1} \neq k_{2}, \\
\mu_{n}\left(i, j, V_{n} t\right)=\mu_{n}^{(0)}(i, j, t)+V_{n}^{-1} h_{0}(i, j, t)(1+o(1)), i, l \in X,
\end{gathered}
$$


where $h_{0}(i, j, t)$ are continuous functions, for any $k \in Y \mu_{n}^{(0)}(i, j, t) \equiv 0$ as $i \epsilon$ $X_{k}, j \notin X_{k}$ and $V_{n} \rightarrow \infty$. This means that $X$ can be divided on non-intersected regions with small transition rates of the order $O\left(1 / V_{n}\right)$ among them.

Denote by $\Pi_{n}(t)$ a Poisson type process switched by $x_{n}(t)$ with rate $q_{n}(i, t)$ at time $t$ in state $i$ and consider its asymptotic behaviour.

Consider for simplicity the quasi-ergodic case. Suppose that at each $k$ uniformly in $u \in[0, T]$ there exist continuous limits $\mu_{n}^{(0)}(i, j, u) \rightarrow \mu_{0}(i, j, u), i, j \in$ $X_{k}, i \neq j$, and at each fixed $u$ an auxiliary homogeneous $M P\left\{x_{0}^{(k)}(t, u), t \geq 0\right\}$ with state space $X_{k}$ and transition rates $\mu_{0}(i, j, u)$ is ergodic with stationary distribution $\rho_{i}^{(k)}(u), i \in X_{k}$. For any $k \in Y, m \in Y, k \neq m$ we introduce the instantaneous average transition rates among regions

$$
\widehat{\lambda}_{0}(k, m, t)=\sum_{i \in X_{k}} \rho_{i}^{(k)}(t) \sum_{l \in X_{m}} h_{0}(i, l, t) .
$$

Let $y(t)$ be a nonhomogeneous $M P$ with state space $Y$ and transition rates at time $t \widehat{\lambda}_{0}(k, m, t), k \neq m$. Suppose that $q_{n}\left(i, V_{n} t\right)=V_{n}^{-1} q_{0}(i, t)(1+o(1))$, where $q_{0}(i, t)$ are continuous functions. Denote $\widehat{q}(k, t)=\sum_{i \in X_{k}} \rho_{i}^{(k)}(t) q_{0}(i, t)$. Let us introduce a consolidated process $\widehat{x}_{n}(t)=k$, if $x_{n}(t) \in X_{k}, t \geq 0$.

Theorem 8.4 At our assumptions the sequence $\left(\widehat{x}_{n}\left(V_{n} t\right), \Pi_{n}\left(V_{n}(t)\right) J\right.$-converges on $[0, T]$ to the process $\left(y(t), \Pi_{0}(t)\right)$, where $\Pi_{0}(t)$ is a Poisson type process switched by $y(t)$ with local rate in the state $k$ at time $t \widehat{q}(k, t)$.

This result can be extended to the case, when regions $X_{k}$ form $V_{n}-S$-sets or in particular monotone structures in the scale of time $V_{n}$.

It is also possible to study the exit time from some subset $\tilde{X}$ which satisfies conditions of the asymptotic consolidation of states (4.1), (4.1). Using the same technique we can represent the exit time from $\widetilde{X}$ as the time of first jump of $\Pi_{n}(t)$ and prove that asymptotically it is equivalent to the time $\tau$ of first jump of $\Pi_{0}(t)$. We mention that $\tau$ is a $\mathrm{PH}$-type random variable.

That means, in complex systems with different orders of transition rates we in general can approximate the distribution of the time of first failure by PH-type distribution.

\subsection{Heavy Traffic Conditions}

In heavy traffic conditions the trajectory of a system is usually non-stable and goes to infinity. We consider the behaviour of a flow of rare events on the trajectory of a switching type system, which satisfies the averaging principle.

Let for each $n>0 \mathcal{F}_{n k}=\left\{\zeta_{n k}(t, x, z), t \geq 0, x \in X, z \in \mathcal{R}^{r}\right\}, \quad k \geq 0$ be jointly independent families of random processes in $D_{\infty}^{r}, x_{n}(t), t \geq 0$ be an 
independent of $\mathcal{F}_{n k}$ semi-Markov process $(S M P)$ in $X$ which plays the role of switching environment, $S_{n 0}$ be the initial value. Denote by $0=t_{n 0}<t_{n 1}<$ $\cdots$ the epochs of sequential jumps of $x_{n}(\cdot)$, and $x_{n k}=x_{n}\left(t_{n k}\right), k \geq 0$. We construct a process with semi-Markov switches (PSMS) in the following way. Let $S_{n k+1}=S_{n k}+\xi_{n k}$, where $\xi_{n k}=\zeta_{n k}\left(\tau_{n k}, x_{n k}, S_{n k}\right), \quad \tau_{n k}=t_{n k+1}-t_{n k}$, and let $\zeta_{n}(t)=S_{n k}+\zeta_{n k}\left(t-t_{n k}, x_{n k}, S_{n k}\right)$ as $t_{n k} \leq t<t_{n k+1}, t \geq 0$. Then the process $\left(x_{n}(t), \zeta_{n}(t)\right), t \geq 0$ is a PSMS (see Anisimov (1994), (1995)).

Let $\left\{q_{n}(x, z), x \in X, z \in \mathcal{R}^{r}\right\}$ be a family of non-negative functions. We construct a Poisson type process $\Pi_{n}(t)$ switched by $\left(x_{n}(t), \zeta_{n}(t)\right)$ as follows: if at time $t x_{n}(t)=x, \zeta_{n}(t)=z$, then $\Pi_{n}(t)$ has the rate of jump $q_{n}(x, z)$.

Consider for simplicity the homogeneous case (distributions of processes $\zeta_{n k}(\cdot)$ do not depend on the index $\left.k \geq 0\right)$. Let $\tau_{n}(x)$ be the sojourn time in state $x$ of $x_{n}(\cdot)$. Denote $\xi_{n}(x, z)=\zeta_{n 1}\left(\tau_{n}(x), x, z\right), \quad g_{n}(x, z)=\sup \left\{\left|\zeta_{n 1}(t, x, z)\right|\right.$ : $\left.t<\tau_{n}(x)\right\}, x \in X, z \in \mathcal{R}^{r}$.

Suppose that an imbedded $M P x_{n k}, k \geq 0$ has at each $n \geq 0$ the stationary measure $\pi_{n}(A), A \in \mathcal{B}_{X}$ and put $m_{n}(x)=\mathbf{E} \tau_{n}(x), b_{n}(x, z)=\mathbf{E} \xi_{n}(x, n z)$, $m_{n}=\int_{X} m_{n}(x) \pi_{n}(\mathrm{~d} x), b_{n}(z)=\int_{X} b_{n}(x, z) \pi_{n}(\mathrm{~d} x), q_{n}(z)=\int_{X} q_{n}(x, n z) \pi_{n}(\mathrm{~d} x)$.

Theorem 8.5 Suppose that $n^{-1} S_{n 0} \stackrel{\mathrm{P}}{\longrightarrow} s_{0}$, there exists a sequence of integers $r_{n}$ such that $n^{-1} r_{n} \rightarrow 0, \sup _{k \geq r_{n}} \varphi_{n}(k, X) \rightarrow 0$, where $\varphi_{n}(k, X)$ is a s.m.c. for $x_{n k}$ (see (2.1)), for any $N>0, \varepsilon>0$

$$
\lim _{n \rightarrow \infty} \sup _{|z|<N} \sup _{x} n \mathbf{P}\left\{n^{-1} g_{n}(x, n z)>\epsilon\right\}=0,
$$

$\limsup _{n \rightarrow \infty} \sup _{|z|<N} \sup _{x}\left\{\mathbf{E} \tau_{n 1}(x) \chi\left(\tau_{n 1}(x)>L\right)+\mathbf{E}\left|\xi_{n 1}(x, n z)\right| \chi(|\xi(x, n z)|>L)\right\} \rightarrow 0$, as $L \rightarrow \infty$, for any $x$ as $\max \left(\left|z_{1}\right|,\left|z_{2}\right|\right)<N \quad\left|b_{n}\left(x, z_{1}\right)-b_{n}\left(x, z_{2}\right)\right|<C_{N} \mid z_{1}-$ $z_{2} \mid+\alpha_{n}(N)$, and $\left|n q_{n}\left(x, n z_{1}\right)-n q_{n}\left(x, n z_{2}\right)\right|<C_{N}\left|z_{1}-z_{2}\right|+\alpha_{n}(N)$, where $C_{N}$ are some constants, $\alpha_{n}(N) \rightarrow 0$ uniformly in $\left|z_{1}\right|<N,\left|z_{2}\right|<N$, also there exist functions $b(z), q(z)$ and a constant $m$ such that for any $z \in \mathcal{R}^{r}$ $b_{n}(z) \rightarrow b(z), n q_{n}(z) \rightarrow q(z), m_{n} \rightarrow m>0$ and $b(z)$ has no more than linear growth.

Then the sequence $\left(n^{-1} \zeta_{n}(n t), \Pi_{n}(n t)\right) J$-converges on $[0, T]$ to the process $\left(s(t), \Pi_{0}(t)\right)$, where $s(0)=s_{0}, \mathrm{~d} s(t)=m^{-1} b(s(t)) \mathrm{d} t$ and $\Pi_{0}(t)$ is a nonhomogeneous Poisson process with local rate at time $t q(s(t))$.

The proof is essentially based on averaging principle type theorems for processes with semi-Markov switches given in Anisimov (1994),(1995).

We mention that if the state space of $x_{n}(t)$ satisfies conditions of asymptotic consolidation of states (see (4.1),(4.2)), then in the limit we get the process $\left(s(t), \Pi_{0}(t)\right)$ which is switched by $M P y(t)$ (see Theorem 8.4). 


\subsection{Analysis of Reliability of Queueing Models}

We consider the basic state-dependent queueing system $M_{S M, Q} / M_{S M, Q} / m / k$ and, basing on the previous results, study different cases devoted to the reliability analysis in light and heavy traffic conditions.

\subsubsection{Light traffic analysis in models with finite capacity}

Consider a nonhomogeneous model $M_{M, Q} / M_{M, Q} / m / k$ switched by some external Markov environment.

Let $x_{n}(t), t \geq 0$ be a nonhomogeneous $M P$ with values in $X=\{1,2, . ., r\}$ and transition rates $c_{n}(i, j, t), i, j \in X, i \neq j, t \geq 0$. Let the family of nonnegative functions $\left\{\lambda_{n}(i, t, q), \mu_{n}(i, t, q), q \geq 0, i \in X, t \geq 0\right\}$ be given. There are $m$ servers and $k$ places for waiting. Denote by $Q_{n}(t)$ the number of calls in the system at time $t$. The system operates in the following way. Calls enter the system one at a time. If at time $t x_{n}(t)=i$ and $Q_{n}(t)=q$, then with rate $\lambda_{n}(i, t, q)$ a call may enter the system and it takes an idle server if there is one. If not, it goes to a queue if there are no more then $k+m-1$ calls in the system. Otherwise this call is lost. A service rate for each busy server is $\mu_{n}(i, t, q)$.

Suppose that we have light traffic and slow dependence on $t$, that is:

$$
\begin{gathered}
\lambda_{n}\left(i, V_{n} t, q\right)=\varepsilon_{n} \lambda_{0}(i, t, q)(1+o(1)), i \in X, \\
\mu_{n}\left(i, V_{n} t, q\right)=\mu_{0}(i, t, q)(1+o(1)), i \in X, \\
c_{n}\left(i, j, V_{n} t\right)=c_{i j}(t)(1+o(1)), i, j \in X, i \neq j,
\end{gathered}
$$

where $\varepsilon_{n} \rightarrow 0, V_{n}=\varepsilon_{n}^{-k-m-1}$, functions $\lambda_{0}(i, t, q), \mu_{0}(i, t, q), c_{i j}(t)$ are continuous, and all values $o(1) \rightarrow 0$ uniformly in $t$ on some interval $[0, T]$.

Consider at each fixed $t$ an auxiliary homogeneous $M P$ with transition rates $c_{i j}(t), i, j \in X$ and suppose that it's ergodic with stationary distribution $\rho_{i}(t), i \in X$. Let $A(t, q)$ and $G(q, t)$ be diagonal matrices with elements $\lambda_{0}(i, t, q)$ and $\min (q, m) \mu_{0}(i, t, q)$ correspondingly, $C(t)=\left\|c_{i j}(t)\right\|, i, j \in X, i \neq j$, where we assume $c_{i i}(t)=-\sum_{j \neq i} c_{i j}(t)$.

Denote by $\bar{\rho}(t)$ and $\overline{1}$ row vectors with elements $\rho_{i}(t)$ and 1 , and put

$$
\widehat{\lambda}(t)=\bar{\rho}(t)\left(\prod_{q=0}^{m+k-1} A(q, t)(G(q+1, t)-C(t))^{-1}\right) A(m+k, t) \overline{1} .
$$

Let $\Omega_{n}(i, s)$ be the time of first loss of a call given that $x_{n}(0)=i, Q_{n}(0)=s$, and $Y_{n}(t)$ be the number of lost calls on the interval $[0, t]$.

Theorem 8.6 If conditions (6.1)-(6.3) are satisfied, then the relation (3.2) is true and $Y_{n}\left(V_{n} t\right) J$-converges to the Poisson Process with local rate $\widehat{\lambda}(t)$. 
In particular if there is no Markov environment then

$$
\widehat{\lambda}(t)=\left(\prod_{q=0}^{m+k-1} \lambda_{0}(q, t) \mu_{0}(q+1, t)^{-1}\right) \lambda_{0}(m+k, t) .
$$

We mention that some classes of Markov and semi-Markov queueing models with fast service in homogeneous case were considered in Anisimov et al. (1987), Anisimov (1996).

Remark 8.4 If $x_{n}(t)$ satisfies conditions of asymptotic consolidation in the scale of time $V_{n}$ (see (4.1),(4.1)), then using Theorem 8.4 we get $J$-convergence of $Y_{n}\left(V_{n} t\right)$ to Poison process $\Pi(t)$ switched by limiting consolidated MP. Correspondingly $V_{n}^{-1} \Omega_{n}(i, s)$ weakly converges to the time of 1 st jump of $\Pi(t)$.

\subsubsection{Heavy traffic analysis}

Consider now the model $M_{S M, Q} / M_{S M, Q} / 1 / \infty$ in heavy traffic conditions. For simplicity we study the homogeneous case and suppose that parameters of the model do not depend on $n$. The system is described by a homogeneous SMP $x(t), t \geq 0$ with values in $X=\{1,2, . ., r\}$. Process $x(t)$ plays a role of environment. Input and service rates are $\lambda(i, z)$ and $\mu(i, z)$ correspondingly, $q \geq 0, i \in X, t \geq 0$. There is one server and infinitely many places for waiting. Denote by $Q(t)$ the number of calls in the system at time $t$.

Suppose that calls are impatient, that is each call independently of others may get a refusal (be lost) with local rate $n^{-1} q\left(x(t), n^{-1} Q(t)\right)$, where $q(i, z)$ is some continuous function. Let $Y_{n}(t)$ be the number of lost calls on the interval $[0, t]$. Suppose that $x(t)$ is ergodic with stationary distribution $\rho_{i}, i \in X$. Denote

$$
\widehat{\lambda}(z)=\sum_{i \in X} \lambda(i, z) \rho_{i}, \widehat{\mu}(z)=\sum_{i \in X} \mu(i, z) \rho_{i}, \widehat{q}(z)=\sum_{i \in X} q(i, z) \rho_{i},
$$

Theorem 8.7 If $Q(0)=n q_{0}$, functions $\lambda(i, z), \mu(i, z), q(i, z)$ are locally Lipschitz with respect to $z$, and the function $b(z)$ has no more then linear growth, then $Y_{n}(n t) J$-converges on $[0, T]$ to the Poisson process with local rate $q(s(t))$, where

$$
s(0)=q_{0}, \quad \mathrm{~d} s(t)=(\widehat{\lambda}(s(t))-\widehat{\mu}(s(t))) \mathrm{d} t,
$$

and $T$ is any positive value such that $s(t)>0$ on the interval $[0, T]$.

The proof uses the result of Theorem 8.5 and the representation of $Q(t)$ as a Switching Process (switching times $t_{1}<t_{2}<\ldots$ are the epochs of jumps of $x(t))$.

Analogous results can be proved for semi-Markov queueing networks. 


\subsubsection{Systems with highly reliable servers}

Consider the system $M_{Q} / M_{Q} / r / \infty$ in heavy traffic conditions. Suppose for simplicity that there is no semi-Markov environment $x(t)$ and there are $r$ servers which are subject to random failures. The system is described by families of functions $\{\lambda(q), \mu(q), q \geq 0\}$ and random variables $\{\eta(q), \kappa(q), q \geq 0\}$. Values $\eta(\cdot)$ correspond to batches (or volumes of information) of input calls, and $\kappa(\cdot)$ correspond to batches of served calls. Suppose that rates of failure and repair for servers are small (of the order $O(1 / n)$ ). That means, if at time $t n^{-1} Q(t)=q$, then the instantaneous rate of failure for each working server is $n^{-1} a(q)$, and the instantaneous rate of repair for each failed server is $n^{-1} c(q)$. In this case we naturally get the model which allows the asymptotic consolidation of states.

Denote by $R_{n}(t)$ the number of servers "on" at time $t$. Let $g(q)=$ $\mathbf{E} \eta(q), v(q)=\mathbf{E} \kappa(i, q)$.

Theorem 8.8 Suppose, that $Q(0)=n q_{0}, R_{n}(0)=r_{0}$, variables $\eta(q), \kappa(q)$ are integrable uniformly in $q$ in any bounded region, functions $\lambda(q), \mu(q), g(q)$, $v(q), a(q), c(q)$ are locally Lipschitz with respect to $q$ and have no more then linear growth. Then the sequence of processes $\left(R_{n}(n t), n^{-1} Q(n t)\right) J$-converges on $[0, T]$ to the MP $(R(t), z(t))$ in $\{0,1, . . r\} \times[0, \infty)$ such that $R(0)=r_{0}$, and at fixed $z(t)=q$ the process $R(t)$ has the following local rates of transitions: the rate from $k$ to $k+1$ is $(r-k) b(q)$; from $k$ to $k-1$ is $k a(q)$, otherwise the rate is zero, $k=0,1, . ., r$. Correspondingly, the process $z(t)$ satisfies the equation:

$$
z(0)=q_{0}, \mathrm{~d} z(t)=(g(z(t)) \lambda(z(t))-R(t) v(z(t)) \mu(z(t))) \mathrm{d} t .
$$

Here $T$ is any positive value such that $z(t)>0$ on $[0, T]$ with probability one.

We mention that the process $R(t)$ at fixed $z(t)=q$ locally behaves as a Birth-and-Death process with rates $(r-k) b(q)$ and $k a(q)$ correspondingly and in some sense it plays the role of environment for $z(t)$. But in this case we have a feedback between the environment and the process itself.

This result can be extended to the case, when we have additional semiMarkov environment, and also to queueing networks $\left(M_{S M, Q} / M_{S M, Q} / k_{i} / \infty\right)^{r}$ with unreliable servers.

\section{References}

1. Anisimov, V.V. (1970). Limit distributions of functionals of a semiMarkov process given on a fixed set of states up to the time of first exit, Soviet Math. Dokl., 11, No. 4, 1002-1004.

2. Anisimov, V.V. (1973). Asymptotic consolidation of the states of random processes, Cybernetics, 9, No. 3, 494-504. 
3. Anisimov, V.V. (1974). Limit theorems for sums of random variables in an array of sequences defined on a subset of states of a Markov chain up to the exit time, Theor. Probability and Math. Stat., No. 4, 15-22.

4. Anisimov, V.V. (1978). Applications of limit theorems for switching processes, Cybernetics, 14, No. 6, 917-929.

5. Anisimov, V.V. (1988). Random Processes with Discrete Component. Limit Theorems, Publ. Kiev Univ., Kiev (Russian).

6. Anisimov, V.V. (1994). Limit theorems for processes with semi-Markov switches and their applications, Random Oper. \& Stoch. Eqv., 2, No. 4, $333-352$.

7. Anisimov, V.V. (1995). Switching processes: averaging principle, diffusion approximation and applications, Acta Applicandae Mathematicae, Kluwer, 40, 95-141.

8. Anisimov, V.V. (1996). Asymptotic analysis of switching queueing systems in conditions of low and heavy loading, Matrix-Analytic Methods in Stochastic Models, Eds. S.R. Chakravarthy and A.S. Alfa, Lect. Notes in Pure and Appl. Mathem. Series, Marcel Dekker, Inc., 183, 241-260,

9. Anisimov, V.V. (1998). Asymptotic Analysis of Stochastic Models of Hierarchic Structure and Applications in Queueing Models, Advances in Matrix Analytic Methods for Stochastic Models, Eds. A. S. Alfa, S. R. Chakravarthy, Notable Publ. Inc., USA, 237-259.

10. Anisimov, V.V. (1999). Diffusion approximation for processes with semiMarkov switches and applications in queueing models, Semi-Markov Models and Applications, Eds. J. Janssen and N. Limnios, Kluwer Academic Publishers, Dordrecht, The Netherlands.

11. Anisimov, V.V. and Aliev, A.O. (1990). Limit theorems for recurrent processes of semi-Markov type, Theor. Probab. \& Math. Statist., No. 41, $7-13$.

12. Anisimov, V.V. and Sztrik, Ja. (1989). Asymptotic analysis of some complex renewable systems operating in random environments, European J. of Operational Research, 41, 162-168.

13. Anisimov, V.V., Zakusilo, O.K. and Dontchenko, V.S. (1987). Elements of Queueing Theory and Asymptotic Analysis of Systems, Publ. "Visca Scola", Kiev (Russian).

14. Bobbio, A. and 'Trivedi, K.S. (1986). An Aggregation technique for the transient analysis of stiff Markov chains, IEEE Trans. on Computers, C-35, 9, 803-814. 
15. Courtois, P.J. (1977). Decomposability: Queueing and Computer Systems Applications, Academic Press, New York.

16. Kovalenko, I.N. (1980). Rare Events Analysis in the Estimation of Systems Efficiency and Reliability, Publ. "Sov. Radio", Moscow (Russian).

17. Kovalenko, I.N. (1994). Rare events in queueing systems, A survey, Queueing Systems, 16, 1-49.

18. Korolyuk, V.S. and Turbin A.F. (1978). Mathematical Foundations of Phase Consolidations of Complex Systems, Publ. "Naukova Dumka", Kiev (Russian).

19. Soloviev A.D. (1970). A redundancy with fast repair, Izv. Acad. Nauk SSSR. Technich. Kibernetika, 1. 56-71 (Russian).

20. Sztrik, Ja. (1992). Asymptotic analysis of a heterogeneous renewable complex system with random environments, Microelectronics and Reliability, 32, 975-986

21. Sztrik, Ja. and Kouvatsos, D. (1991). Asymptotic analysis of a heterogeneous multiprocessor system in a randomly changing environment, IEEE Trans. on Software Engineering, 17, No. 10, 1069-1075. 